\title{
Type Design and Behavior Control for Six Legged Robots
}

\author{
Ling Fang and Feng Gao*
}

\begin{abstract}
The research on legged robots attracted much attention both from the academia and industry. Legged robots are multi-input multi-output with multiple end-effector systems. Therefore, the mechanical design and control framework are challenging issues. This paper reviews the development of type synthesis and behavior control on legged robots; introduces the hexapod robots developed in our research group based on the proposed type synthesis method. The control framework for legged robots includes data driven layer, robot behavior layer and robot execution layer. Each layer consists several components which are explained in details. Finally, various experiments were conducted on several hexapod robots. The summarization of the type synthesis and behavior control design constructed in this paper would provide a unified platform for communications and references for future advancement for legged robots.
\end{abstract}

Keywords: Legged robot, Type synthesis, Human robot interaction, Control framework

\section{Introduction}

Since the last decade, research on legged robots has gained much attention, with its development represents the state of art technology in both industry and research field [1]. The evolvement of legged robots has progressed rapidly. Many companies and universities designed various type of autonomous robots. One of the outstanding organizations is Boston Dynamics, which created two and four legged robots, including BigDog, Atlas and LS3 [2-4]. In addition, six legged robots were developed, such as Athlete (NASA), Whegs (Quinn) and Octopus (SJTU) [5-7]. With mainstream media converges on BigDog, SpotMini, Handle, etc., people are demanded for legged robots that can operate in unstructured environment and accomplish tasks to assist human.

Unlike industry arm robots which are multi-input multi-output system with single end-effector, legged robots are multi-input multi-output with multiple endeffector systems with nonlinear mapping between input and output [8]. Therefore, legged robots are much more challenging and complicated to design and control.

*Correspondence: fengg@sjtu.edu.cn

State Key Laboratory of Mechanical System and Vibration, Shanghai Jiao Tong University, Shanghai 200240, China
One of the major advantages of legged robot is their flexibility in adapting to complicated environments. Hence, they are commonly applied in unstructured settings. These robots can even overcome terrains with considerable variations and spatial constraints [9-12]. Moreover, legged robots are often capable of integrating walking and operational tasks because of high degree of freedoms (DoF) on their body frame [13].

Despite of many prototypes of legged robots are designed, very few are utilized to facilitate human daily life or mass industry operations [14]. There are two main reasons which cause this drawback. Firstly, given a specific task or application requirement to the robots, the design of the legged robots is not mathematically associated with the demand [15]. Secondly, the control system is very complicated with little high-level guidance [16]. Because of these two challenges, this paper focuses on the following two questions: How to design the mechanisms of legged robots according to certain application requirements? How to design the control system of the legged robots to achieve autonomy?

In this paper, the development of type synthesis and behavior control system are firstly reviewed. The mechanical design on various type synthesis model are demonstrated. With each type synthesis model, a design prototype of a six-legged robot is provided. Three layers, 
including data driven layer, robot behavior layer and robot execution layer of the control framework, are introduced. Each layer consists of several components. Finally, various complicated tasks conducted by several hexapod robots are introduced and discussed.

\section{Review on Type Synthesis and Control Framework of Legged Robots}

The design of mechanical system consists of structure and mechanism components [17]. The mechanism design contains three important aspects: characteristic evaluation, dimension synthesis and type synthesis. Among these three, type synthesis is the most original and inventive one [18]. Various mathematical tools were used in the type synthesis to solve the problem of setting up type criteria with classifications of design objectives [19-22]. Type criteria is not just the extension of the dimensions or description of end-effector motion. It has to express the end-effector characteristics completely and precisely, with concern on the succession of motions and the interactions between different motion attributes [18].

As the theoretical foundation of type synthesis, the mathematical theory is the tool to establish type criteria at the same time to classify and describe mechanisms. Appropriate mathematical tools can systemize the complicated synthesis process. The integrity of type criteria determines the integrity of mechanisms classifications and the correctness of the type synthesis results. At present, several kinds of mathematical tools are utilized in different methodologies, such as the screw theory [23], the theory of differential geometry including the Lie group and differential manifolds [21], the theory of linear transformation and the set theory [24]. A number of researchers, including Mruthyunjaya [19], Huang et al. [5, 25, 26], Herv [21], Gogu [27, 28], Jin et al. [29], Shen et al. [30], Meng et al. [31], Gao et al. [24, 32-34], Gan et al. [20], and $\mathrm{Bi}$ et al. [22], contributed much to the development of the type synthesis of parallel and serial mechanism.

The screw is composed of two vectors, expressing the direction and the position of the screw axis, respectively. The motion and the constraint screw systems are reciprocal systems, which are useful to replace the complex intersection operation of screws with the simple union operation [35]. The motion system of a limb can be transformed into the corresponding constraint system. The union of all limbs' constraint systems is the constraint system of the parallel mechanism's mobile platform, of which reciprocal system is the motion system of the mobile platform $[5,26]$. The screw theory is an effective tool to analyze the instantaneous motions of mechanisms [36].
Based on the theory of differential geometry, the concepts of differential manifolds, displacement submanifolds, Lie groups and displacement subgroups are utilized to derive type synthesis [21]. The motion characteristics, which fulfill the algebra structures of the Lie group, are represented by twelve kinds of displacement subgroups. Those subgroups are not influenced by the succession of motions. Other motion characteristics, which do not fulfill the algebra structures of the Lie group, are represented by displacement submanifolds. Those submanifolds are sensitive to the succession of motions. The Lie groups and differential manifolds provided precise mathematical descriptions for the motion characteristics of kinematic pairs and chains, laying the foundation for kinematic analysis and type synthesis [31,37].

Position and orientation characteristic (POC) sets were proposed upon the output translational and rotational velocities of mechanical end-effectors [38, 39]. POC sets contain fifteen separate kinds and they are breakthrough by focusing on the characteristics of end-effectors rather than separate kinematic pairs [30].

The generalized function $\left(\mathrm{G}_{F}\right)$ sets are the special sets of motion elements of mechanical end-effectors [40]. Taking the succession and the interaction of motion characteristics into account, the $\mathrm{G}_{F}$ sets represent the motion ability of end-effectors. The type synthesis approach based on the $\mathrm{G}_{F}$ sets is developed by combining the intersection algorithms and the number synthesis formulas [24, 32-34].

A considerable number of researchers developed distinct methodologies for the type synthesis problem. As a result, this field is undergoing significant progress, which can be ascertained from the considerable amount of publications on various journals and conference proceedings. However, type synthesis for legged robots are not the same as parallel or serial system. Hence, the methods mentioned above cannot be utilized directly for designing legged robots. Unlike parallel or serial mechanisms, which usually have single end-effector (manipulator), legged robots normally possess multiple end-effectors, including body (main output), legs and hands. A type synthesis method that can describe the motion of the legs and the body for legged robots is still in need. How to express and classify the output of end-effectors are challenging. The commonly designed body of legged robots are three, four, five or six dimensions. A proper type synthesis model should consider all of the above output dimensions.

Not only the type synthesis for legged robots is important, the control the robots is equally crucial. The control architecture of robots mainly includes four models: sense-plan-act (SPA) architecture, the 
layered architecture, the subsumption architecture and the hybrid architecture [1].

In 1960, Stanford University developed "Shakey", the first robot to deploy a SPA architecture [41, 42]. Based on the initial and the target status, The SPA architecture plans series of sequences of actions [43]. The method is mostly used in the field of machine manufacture to accomplish repetitive and monotonous tasks, such as arm operation and workpiece handling. However, the SPA architecture provides poor maneuverability and flexibility when facing complicated tasks, commands in changing environments.

In 1983, Saridis, a famous scholar in the field of intelligent control, proposed a three-layer model, namely, executive level, coordination level and organizational level [44]. This model is regarded as the most representative framework among the layered architectures. It usually consists of one or more main controllers and many nodes, both of which have processing capacity. This architectural pattern was later applied to NASREM structures proposed by NASA and the U.S. National Bureau of Standards [45]. Layered architecture has a wide range of applications depending on different tasks, environments and the robots' capability [46].

In 1986, Brooks proposed a behavior based architecture "subsumption architecture" from the standpoint of studying the structure of a mobile robot control system $[47,48]$. It is based on behavioral control by decomposing complex tasks into a series of relatively simple and specific sub-behaviors. The subsumption architecture was later widely used, including the intelligent control of spacecraft in the United States, mountainous terrain specialized climbing robot and other projects $[49,50]$. The subsumption architecture is less flexible for multiple tasks in unstructured and unknown environments.

In 1998, Gat proposed one of the most representative hybrid architectures [51]. Usually hybrid architecture for decision-making system has two states, one is the planning oriented; the other is behavior oriented [1]. Often, the top layer of hybrid architecture utilizes the programcontrolled architecture for decision-making to achieve better performance and efficiency. The bottom layer utilizes subsumption architecture to achieve improvement in surrounding adaptability, robustness and real-time performance [48].

The control of legged robots is complicated and challenging because they are multi-input multi-output with multiple end-effectors systems. Furthermore, there is no direct mapping between the task that is given to the robots and the output behavior of the robots. In order to achieve intelligent, full autonomous control or sole human control are not suitable for current stage of legged robots. Therefore, the best control architecture is human-robot interaction with self-regulated autonomy (HRISRA).

Many human-robot interaction (HRI) models utilize force sensors, touch sensors, voice commands and visual information [52-54]. Recently, the development of machine learning, particularly deep learning and big data analysis, promotes general artificial intelligence in robotics [55-58]. Several research results were published on Nature and Science, which are related to autonomous control [59-61]. However, for the legged robots, the mapping between input and output are highly complicated. Currently, few control models are applicable to them. In order to achieve HRISRA, the control framework needs to consider the high level tasks, commands and perception that are given from the human or observed from the environment. In addition, the framework should take the low level robot behaviors such as end effector topology, motion characteristics and trajectory into consideration. Therefore, there is high demand for a control architecture which can achieve HRISRA for legged robots.

The control system of legged robots consists of three layers as shown in Figure 1. The lower layer is execution layer, which is usually based on Linux for real time control purpose [62]. The middle layer is communication layer, which could be based on Robot Operating System (ROS) [63]. The top layer is a "brain-like" robot operating system (BROS) which serves as a high level control system to deal with tasks, instructions and sensor fusion processing. Currently there is no mature top layer control. The establishment of BROS system will play a key role for legged robot HRISRA control. The goal of BROS system is to reveal the association between "Task - Command - Perception" and end effector's "Topology - Motion -Trajectory" for legged robots.

The construction of BROS confronts the challenges of various tasks, commands and environments that change over time. In addition, several conflicts should be resolved: human command versus autonomous

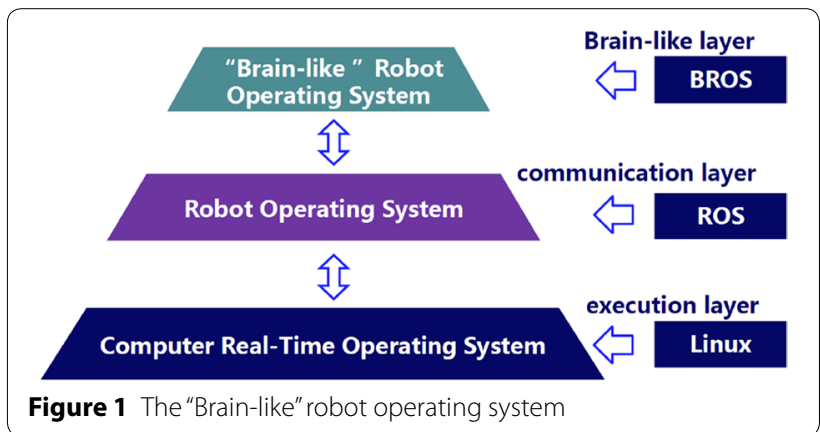


control, human command versus environment information, human command versus the given task, human command versus robot ability.

The development of legged robot control framework has come a long way. The event that brought world attention is DRAPRA Robotics Competition [64]. Particularly, after the Fukushima nuclear accident in Japan, the U.S. Defense Department held the DRAPA Robotics Competition for emergency rescue by conducting the following tasks: driving car, get off the car, open and close the door, screw the valve, break the wall, plug the power, clear obstacles, climb up and down the stairs [64]. It is noteworthy that all the participating teams are using HRI control [65].

Teams competing in the DARPA Finals exhibited one or more of the following HRI characteristics [65]: (1) The robot had more autonomy when performing simpler manipulation and mobility tasks; (2) The operators had more interaction performing complex manipulation and mobility tasks; (3) More models are placed manually to assist robots in performing complicated manipulation tasks; (4) Operators were well trained with ample practice and more than one operator split responsibilities in task executions.

After the competition, a comprehensive analysis review published in International Journal of Robotics Research (IJRR) summarized the experience and lessons learned in the DRAPA competition [65]. The paper concludes that the state of autonomous control in robotics was far from sufficient to support effective teleoperation when completing complex tasks. One of the most critical issues encountered in HRI is to achieve the right balance between human supervision and robot autonomy. Effective interaction should balance robot autonomy with the skills and capabilities that humans are superior at. These skills include decision making, perceptual capabilities, strategic thinking, and overall task awareness [66]. Therefore, HRI control framework allows the robot focus on low-level tasks such as terrain transversal, while maintaining the highlevel control from human.

Designing the control framework for legged robots, still faces many issues: (1) How to balance between human supervision and robot autonomy? (2) How to decompose complex tasks into a sequence of subtasks by forming a subtask chain? (3) How to mathematically express the robot behaviors including the topology, the end-effector motion and trajectory characteristics by forming a sequence of behavior chains? (4) How to establish the relationship between subtask chains and behavior chains? The research of answering these basic questions helps the advancement of the field. Therefore, it is worthwhile to find the proper models to the issues mentioned above.

\section{Type Synthesis of Legged Robots Based on $G_{F}$ Sets}

The type criteria, as the design objectives of the type synthesis, should be precise in describing the motion characteristics of end-effectors and providing complete classifications $[67,68]$. In order for type synthesis to be utilized in the designing of legged robots, the type criteria should be non-algebraic and dimensionless, independent of coordinate systems and endowed with physical meanings. Therefore, the type criteria could be a set of several elements, which represent the characteristics of endeffectors with succession [69].

One of the mathematical tool that can describe the end-effectors characteristics of legged robot is the generalized function $\left(G_{F}\right)$ sets $[24,32,34,70]$. The fundamental elements of the end-effectors characteristics space include three-dimensional translations $\left(T_{a}, T_{b}, T_{c}\right)$ and three-dimensional rotations $\left(R_{\alpha}, R_{\beta}, R_{\gamma}\right)$. The three translation axes $a, b, c$ are non-coplanar simultaneously and two of them are not collinear. The three rotation axes $\alpha$, $\beta, \gamma$ always intersect at a common point, not coplanar simultaneously and two of them are not collinear. $R_{\gamma}$ is the last rotation axis relative to the middle rotation axis $R_{\beta}$, which is relative to the base rotation axis $R_{\alpha}$.

The fundamental elements in $\mathrm{G}_{F}$ sets express the types, quantities, succession, and completeness of elements. The six-dimensional universal set is represented by $\mathrm{G}_{F}$ $\left(T_{a}, T_{b}, T_{c}, R_{\alpha}, R_{\beta}, R_{\gamma}\right)$. The elements represent the existence of end-effectors characteristics. They are non-algebraic, dimensionless, and independent of the choice of coordinate systems. Therefore, the $\mathrm{G}_{F}$ sets are more suitable for the type synthesis of legged robots.

The $G_{F}$ sets are classified into three categories and twenty-five types in total [71]. The first category of $\mathrm{G}_{F}$ sets is to represent mechanisms of end-effectors that contain complete rotation $\left(R_{\alpha}, R_{\beta}, R_{\gamma}\right)$ in all configurations. The second category is to represent the end-effectors that have none complete rotation characteristics in all configurations. The third category is for the end-effectors containing two or three dimensional rotation characteristics and only one of which is complete among any configuration. Among these 25 types of $G_{F}$ sets, 9 types of them are suitable for designing legged robots as shown in Table 1. The first column shows the degree of freedom (DoF) of a given end effector. The second column shows the characteristics of the end-effector with $\mathrm{G}_{F}$ sets notion. The last column provides images to demonstrate the motion of the end-effectors.

Our research group developed several hexapod robots based on different type synthesis categories shown in Table 1 . The first robot shown in Figure 2 is an isotropic hexapod robot driven by 18 actuators. Each leg of the robot is a 3-DoF parallel mechanism 
Table $1 G_{F}$ sets of end-effector characteristics suitable for legged robots

\begin{tabular}{lll}
\hline DoF & Characteristics of end-effector & End-effector description \\
\hline $6 \quad G_{F 1}^{\prime}\left(T_{a}, T_{b}, T_{C}, R_{\alpha}, R_{\beta}, R_{\gamma}\right)$ & $G_{F}^{I I I}\left(R_{\alpha}, T_{a}, T_{b}, R_{\beta}, R_{\gamma}, 0\right)$
\end{tabular}

$4 \quad G_{F 3}^{\prime}\left(T_{a}, T_{b}, T_{c}, R_{\alpha}, 0,0\right)$

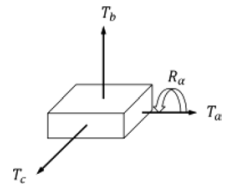

$G_{F 25}^{\prime \prime \prime}\left(R_{\beta}, T_{a}, T_{b}, R_{\gamma}, 0,0\right)$

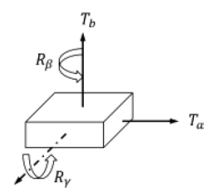

3

$G_{F 4}^{\prime}\left(T_{a}, T_{b}, T_{c}, 0,0,0\right)$

$G_{F 5}^{\prime}\left(T_{a}, T_{c}, R_{\beta}, 0,0,0\right)$

$G_{F 16}^{\prime \prime}\left(R_{\beta}, T_{a}, T_{b}, 0,0,0\right)$
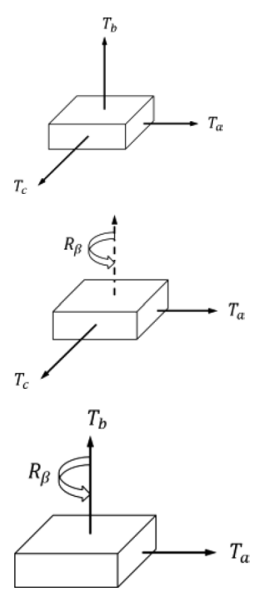

$2 \quad G_{F 19}^{\prime \prime}\left(R_{\beta}, T_{a}, 0,0,0,0\right)$ $T_{a}$ and $R_{\beta}$ are linearly independent

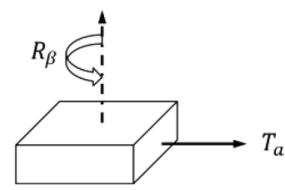

1.5 $G_{F 19}^{\prime \prime}\left(R_{\beta}, T_{a}, 0,0,0,0\right)$ $T_{a}$ and $R_{\beta}$ are not independent

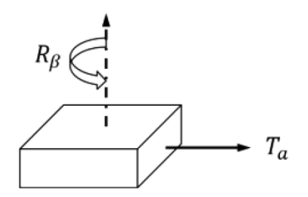

and the actuation can be controlled both by position and force. The characteristics of body movement is $G_{F}^{I}\left(T_{a}, T_{b}, T_{c}, R_{\alpha}, R_{\beta}, R_{\gamma}\right)$. All the legs are distributed evenly around the body. In a single leg, the ankle is connected to the body via 3 limbs: 1 UP and 2 UPS.

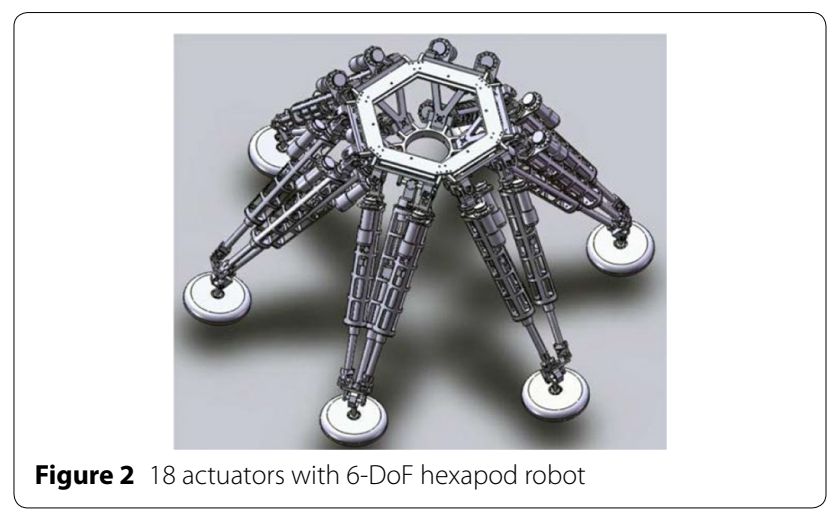

Another spherical joint is added between the foot and the ankle for the adaption of the uneven ground. The robot body can move with 6 DoF similar to Stewart machine.

The 18 actuators with 6-DoF parallel hexapod robot derives different types of robots by varying the length of the legs, the size of the body, the manipulators mounted on the body and the sensors mounted on the legged robots. Several robots were developed and shown in Figure 3 , including dexterous operating robot, heavy load shipping robot, heavy load precision operating robot, heavy load operating robot, fire rescue robot and heavy load dexterous operating robot.

Besides the isotropic hexapod robot, other hexapod robots with less actuators are also developed shown in Figure 4. These robots can also achieve tasks with heavy loading capacity. The reduction of actuator has the following advantages: (1) Reducing the cost: the main expenses of the robot come from the actuation system, hence less input means less cost. (2) Reducing the weight and the size of the robot. (3) Improving the reliability of the robot: the less of the actuators, the lower probability of malfunction. (4) Improving the battery life; (5) More flexibility in designing the structure of legs and the body to adapt to different tasks and environments; (6) Simpler control framework.

One of robots with reduced actuators is a 5 DoF hexapod robot driven by five actuators as shown in Figure 4 . The six legs form two groups. Each group consists of three non-adjacent legs and is driven by two actuators. Among the two actuators, one is utilized to adjust the height of the legs, and the other is to power the legs to move forward. The robot is suitable for crossing ditch and climbing stairs by adjusting the length and height of the legs. The topological gait of this robot is 3-3, that is, the robot can only lift the legs group by group. The fifth actuator mounted on the waist is responsible for the turning. Therefore, its body comes with 3 DoF, 2 translational and 1 rotational. The characteristics of body movement is $G_{F 16}^{I I}\left(R_{\alpha}, T_{a}, T_{b}, 0,0,0\right)$. 


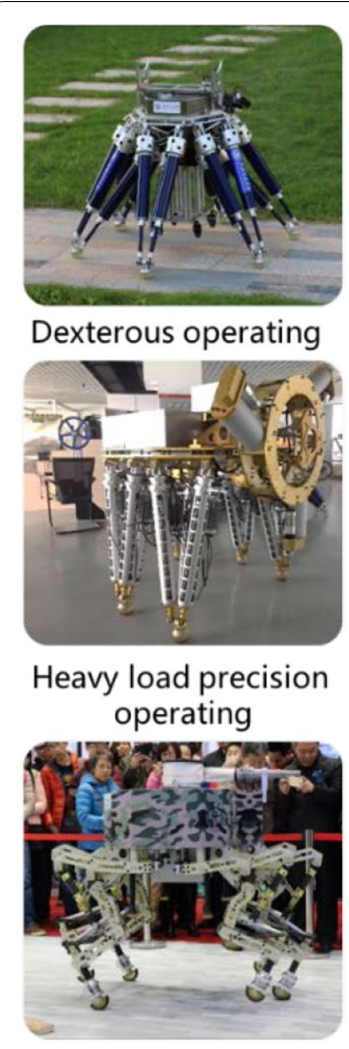

Fire rescue
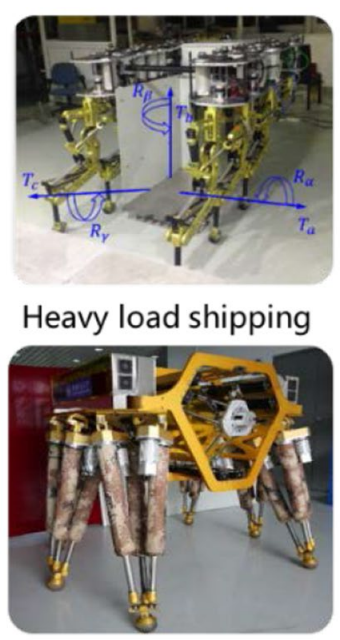

Heavy load operating

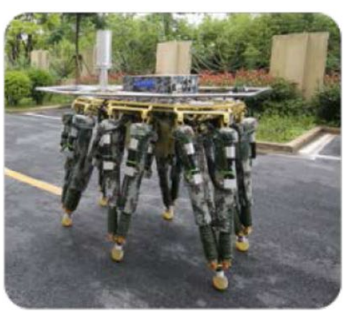

Heavy load dexterous operating
Figure 3 Several types of hexapod robots are designed according to different tasks

The 13 DoF hexapod is driven by 13 actuators shown in Figure 4. Each leg is driven by 2 actuators, one is utilized to move forward and the other is to adjust the height of the leg. Similar to the 5 DoF robot, the 13th actuator is mounted on the body by providing the robot with turning ability. Hence, the robot body can move with 4 DoF, 2 translational and 2 rotational. The characteristics of body movement is $G_{F 25}^{I I I}\left(R_{\alpha}, T_{a}, T_{b}, R_{\beta}, 0,0\right)$.

The 14 DoF hexapod robot derives from the 13 DoF robot. The shell is mounted on the body of the $13 \mathrm{DoF}$ robot. The head, which is a part of the shell, comes with 1 actuator. The head can be adjusted to grab objects, serving as an operational arm. The robot body can move with 5 DoF, 2 translational and 3 rotational. The characteristics of body movement is $G_{F}^{I I I}\left(R_{\alpha}, T_{a}, T_{b}, R_{\beta}, R_{\gamma}, 0\right)$.

Meanwhile, a 3 DoF hexapod robot is still under development. The structure is similar to the 5 DoF robot, but each leg group is driven by 1 actuator, which controls the robot to move forward. Hence there is no height adjustment of the legs. As a result, the robot is not suitable for crossing the ditches or climbing stairs. The robot body can move with 2 DoF, 1 translational and 1

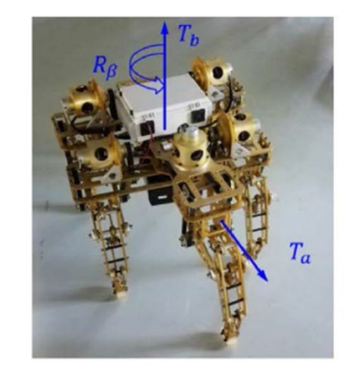

5 DoF

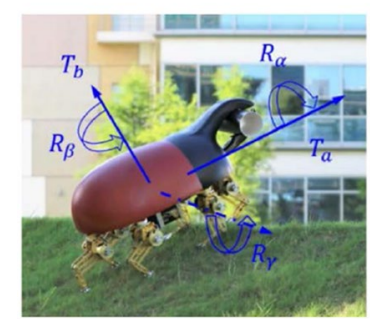

14 DoF

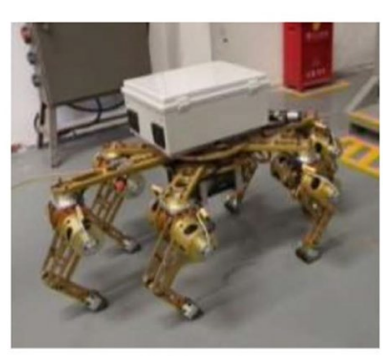

13 DoF

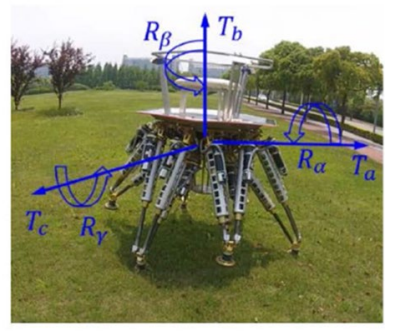

18 DoF
Figure 4 Different types of hexapod robots according to the number of inputs

rotational. The characteristics of body movement is $G_{F 19}^{I I}\left(R_{\alpha}, T_{a}, 0,0,0,0\right)$.

Based on the body movement characteristics shown in Table 1 , the $G_{F}$ sets can be utilized to classify and describe the body movements of existing vehicles. Since the two DoF of a typical car are coupled (1 translational and 1 rotational), the car has $1.5 \mathrm{DoF}$ and its body movement is characterized as $G_{F 19}^{I I}\left(R_{\alpha}, T_{a}, 0,0,0,0\right)$. For AGV cars, two-wheeled vehicles, tanks which utilize the Mecanum wheel, their rotation and translation are decoupled. Therefore, their body has 2 DoFs and the movement is characterized as $G_{F 19}^{I I}\left(R_{\alpha}, T_{a}, 0,0,0,0\right)$.

\section{Control Framework of Legged Robots}

Currently, few control frameworks are designed for legged robots. In order to achieve intelligent control, the framework should include the data driven layer which consists of high level tasks, perception of the environment and human given commands. Furthermore, the control framework should contain robot behavior layer by modeling the robot's end effector topologies, the motion characteristics and trajectories. Finally, the mapping between data driven layer "task-commandperception" and robot behavior layer "topology-motion characteristics-trajectory" should be studied in the framework.

To illustrate the legged robots control framework in details, Figure 5 shows the data driven layer, the robot behavior layer and the actuators execution layer. Each 

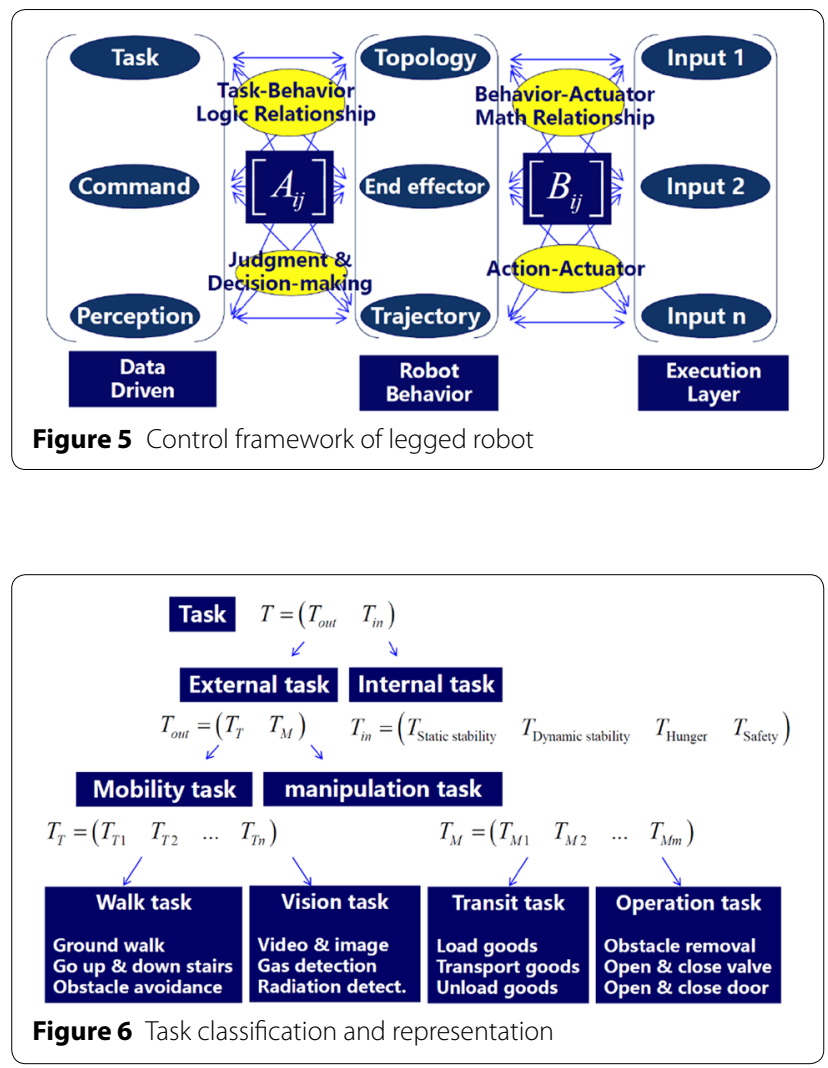

layer consists several building blocks. One of the challenging tasks is to find the mapping between two layers. For instance, matrix A encodes task-behavior logical mapping and matrix $\mathrm{B}$ expresses the behavior-actuator mapping. The rest of this section will focus on the three key components in data driven layer and three key components in robot behavior layer and the mapping between them.

\subsection{Three Components in Data Driven Layer \\ 4.1.1 Task Component}

In unstructured environment, the legged robots needs to accomplish various tasks. Figure 6 illustrates the division of tasks space, including external and internal tasks. The mobility and manipulation tasks are part of the external tasks; static stability, dynamic stability and safety belong to the internal tasks.

There is a continuing need for research to mathematically model different tasks, such as expressing the external tasks as a sequence of subtasks or task chains. For internal task, overturning resistance, shock resistance, disturbance resistance and anti-collision should be considered and mathematical formulation are required.
Table 2 Single actions based on human command

\begin{tabular}{|c|c|c|c|}
\hline \multirow[t]{3}{*}{ Direction } & \multicolumn{3}{|l|}{ Action } \\
\hline & \multicolumn{3}{|c|}{ Single action classification } \\
\hline & $\begin{array}{l}\text { Body translation } \\
\text { length }\end{array}$ & $\begin{array}{l}\text { Body rotation } \\
\text { angle }\end{array}$ & Turning angle \\
\hline Front & $\sqrt{ }$ & $\sqrt{ }$ & \\
\hline Back & $\sqrt{ }$ & $\sqrt{ }$ & \\
\hline Left & $\sqrt{ }$ & $\sqrt{ }$ & $\sqrt{ }$ \\
\hline Right & $\sqrt{ }$ & $\sqrt{ }$ & $\sqrt{ }$ \\
\hline Left front & $\sqrt{ }$ & $\sqrt{ }$ & \\
\hline Right front & $\sqrt{ }$ & $\sqrt{ }$ & \\
\hline Left back & $\sqrt{ }$ & $\sqrt{ }$ & \\
\hline Right back & $\sqrt{ }$ & $\sqrt{ }$ & \\
\hline Up & $\sqrt{ }$ & & \\
\hline Down & $\sqrt{ }$ & & \\
\hline
\end{tabular}

\subsubsection{Human Command Component}

Human-robot interaction commands could be expressed by speech, gesture, force feedback and body language, etc. The goal of interactive expression has to be simple enough for the robots to understand and to be integrated to their actions. For example, Table 2 shows a list of single action commands. When given a legged robot as shown in Figure 2 with 18 actuators, human commands can be expressed in a sequence of actions shown in Table 2. For instance, the command can be in the form of "walk forward for $5 \mathrm{~m}$, turn left for $30^{\circ}$, go up for $5 \mathrm{~m}$. In Table 2, the actions include forward, backward, up, down, left and right. If the robot has high DoF, it could also achieve more complex single actions such as forward left, backward right, etc.

\subsubsection{Perception Component}

The advancement on various sensors progressed the development of legged robots. The perception information collected from the sensors should be interpreted by the robot. An even more challenging task is to integrate different sensor information and learn the suitable model when there is disagreement.

The perception information is diverse and complex. There are two type of perceptions, external and internal. For external perception, the close range vision sensors help the robot for gait planning while the long range sensor can assist in creating 3-D grid map. The force perception can assist walking in unstructured terrain. In addition, the force sensors are crucial in creating a safety workspace for legged robots when interacting with human. The internal perception mainly describes 
the kinematics and dynamics parameters of the robots. Those sensors include gyroscopes, accelerometers, motor encoder and motor current, measuring the robot position, velocity, acceleration. The mathematical description of these information serves as constraint condition for motion planning.

\subsection{Three Components in Robot Behavior Layer}

The behavior of legged robot is reflected by its leg movement, body motion and hand (manipulator) action. Unlike wheeled robot, the varieties in the combination of those motion types pose a challenging task. Hence the key to design useful control framework for six legged robot is providing mathematical tools to describe the motion and movement of the legs, body and manipulator. In addition, the description should be simple enough to be implemented. The following three aspects will be explained: end effectors topology, motion characteristics and trajectory.

\subsubsection{End Effector Topology}

Topology is the top layer of legged robot behavior control and directly associates with the tasks. The design of topology is crucial in dealing with different payloads under unstructured environments. Currently, how different topologies can be connected based on sensor inputs and environments needs further study.

For instance, a hexapod robot has seven types of topologies. Table 3 shows different types of topologies by including $[0,1,2, \ldots, 6]$ legs lifted simultaneous. $M_{T_{0-6}}$ defines the topology when no leg is lifted. This topology is particularly useful for adjusting the overall posture of the body during operation. It can also be utilized when transiting from one topology to another, such as from $M_{T_{3-3}}$ to $M_{T_{2-4}}$. $M_{T_{1-5}}$ defines one leg is lifted and the other five are on the ground. It is suitable for tough environments and heavy loading conditions. The characteristic of the motion generated by topology $M_{T_{1-5}}$ is slow and stable. $M_{T_{2-4}}$ is the topology when two legs are lifted and the other four are on the ground. It is useful in crossing

\section{Table 3 Topology expression and classification}

\begin{tabular}{lll}
\hline Formula & Configuration & Representation \\
\hline$M_{T_{0-6}}$ & $0-6$ & No leg lifted \\
$M_{T_{1-5}}$ & $1-5$ & 1 leg lifted \\
$M_{T_{2-4}}$ & $2-4$ & 2 legs lifted \\
$M_{T_{3-3}}$ & $3-3$ & 3 legs lifted \\
$M_{T_{4-2}}$ & $4-2$ & 4 legs lifted \\
$M_{T_{5-1}}$ & $5-1$ & 5 legs lifted \\
$M_{T_{6-0}}$ & $6-0$ & 6 legs lifted \\
\hline
\end{tabular}

ditches, climbing stairs and slopes, etc. Compared with $M_{T_{1-5}}, M_{T_{2-4}}$ is faster but less stable. $M_{T_{3-3}}$ represents three nonadjacent legs are lifted simultaneously and is the most popular topology for hexapod robots. It is the fastest configuration for walking. $M_{T_{4-2}}, M_{T_{5-1}}$ and $M_{T_{6-0}}$ can be executed as running and jumping configurations. When one or two legs are on the ground, the hexapod robot will lose its stability and have a high tendency of falling. Hence the control of these three topologies are more challenging.

\subsubsection{End-effector Motion Characteristics}

The middle component of the robot behavior layer encodes the motion characteristics of the end-effectors. This component is particularly important for guiding the low level trajectory planning process. For instance, with 18 motors as shown in Figure 2, the legs, the body and the manipulators of an isotropic six-legged robot have six DoFs. If the task given to the robot is to walk through a narrow passage, the robot needs to rotate its body (just like how a human would rotate our body by facing sideways to pass a narrow passage). Hence planning endeffector motion characteristics can facilitate trajectory planning.

Generally, the low-level planning has been established to describe the end-effector's kinematic mobility, including the workspace, velocity, acceleration, payload, stiffness, etc., all of which are in algebraic form with units. These parameters are related to coordinate systems and cannot handle the diverse range of topology performances of the body of the legged robot. In order to describe the motion of the body and end-effectors over time, the $\mathrm{G}_{F}$ sets can be utilized.

\subsubsection{End Effector Trajectory}

End effector trajectory can be classified as manipulator, body and foot trajectories. All of them can be expressed by fifth-degree or seven-degree polynomials, according to the parameters shown in Table 4. If the position, velocity and acceleration in the start point and end point are known, fifth-degree polynomial can be utilized to express trajectory. Besides the above 6 parameters, if jerk is also considered, seven-degree polynomial is more suitable.

The trajectories can also be designed with simpler function such as triangular, rectangular, trapezoidal and elliptical trajectories. The choice of the trajectory depends on different terrains and tasks. Table 4 shows how foot tip trajectory relates to terrain and speed adaption when given various tasks and environments. The second column shows the expression for a given trajectory. 
Table 4 End-effector trajectories

\begin{tabular}{|c|c|c|}
\hline Trajectory type & Expression & Terrain/speed/parameters \\
\hline Triangle & $\operatorname{Tr} j_{F_{-}} \operatorname{Tri}=\operatorname{Trj}(L, H)$ & Flat terrain, low speed \\
\hline Rectangle & $\operatorname{Tr} j_{F_{-} \operatorname{Rec}}=\operatorname{Trj}(L, H)$ & Difficult terrain, low speed \\
\hline Trapezoid & $\operatorname{Trj} j_{-} \operatorname{Tra}=\operatorname{Trj}(L, W, H)$ & Flat terrain, middle speed \\
\hline Oval & $\operatorname{Trj}_{F_{\text {EE Ellipse }}}=\operatorname{Trj}(a, b)$ & Flat terrain, high speed \\
\hline 5th order polynomial & $\operatorname{Trj}(t)=c_{0}+c_{1} t+\cdots+c_{5} t^{5}$ & 2 points of position, velocity, acceleration \\
\hline 7th order polynomial & $\operatorname{Trj}(t)=c_{0}+c_{1} t+\cdots+c_{7} t^{7}$ & 2 points of position, velocity, acceleration, jerk \\
\hline
\end{tabular}

\subsection{Logical Relationship between Data-driven Layer and Robot Behavior Layer}

The modeling and learning of the logical relationship between two layers is a challenging issue in legged robot control. As illustrated in Figure 5, the connection matrix $\mathrm{A}$ indicates the logical mapping between datadriven layer and robot behavior layer. The matrix $\mathrm{B}$ indicates the logical mapping between robot-behavior layer and execution layer. The learning of the elements in $A$ and $B$ depends on the mathematical representation of each layer and the training process.

The task, human command and robot perception form a complicated high dimensional space on the data driven layer. The mathematical models that can discretize each component are described in previous sections. If enough data is collected, a knowledge database is needed for further learning. Constructing a sequence of tasks, human commands and perceptions chain can facilitate the process of finding the mapping of consecutive layers. Given a temporal window, in the robot behavior layer, a sequence of end-effector topologies, motion characteristics and trajectories are formed. The goal is to find the logical mapping for the chains formed in the two layers.

The elements in the logical mapping can be expressed as adjacency matrix, directed graph or a classification model. All of the mappings require a large dataset to train on. This is crucial in building an intelligent control system for legged robot. Therefore, much work should be put into data collection and construction that allows the robot to explore, build and train itself on. Similar to AlphoGo [72], the utilization of large data, computational power and self-learning capability are the keys to build such a knowledge database for legged robots.

\section{Experiments on Hexapod Robots}

Hexapod robot possesses the capability to finish various complicated tasks in unstructured environments. Based on our mechanical and control design, several hexapod robots are developed. The following examples demonstrate their capabilities in execution of complicated tasks.

\subsection{Climbing Stairs}

One of the advantages of the legged robot is its flexibility in climbing stairs. During stair climbing process, a hexapod robot may utilize different topologies, depending on the slope, height, payload and other factors. During the starting phase and ending phase, the robot sometimes need to use different topologies when encountering steep stairs. As shown in Figure 7, before the robot starts climbing, it walks on the flat ground by utilizing $M_{T_{3-3}}$ for speed. When it starts to climb the stairs, it utilizes different topologies in different stages. $M_{T_{0-6}}$ is used to adjust the posture of body without lifting legs, $M_{T_{1-5}}, M_{T_{2-4}}$ and $M_{T_{3-3}}$ are utilized for climbing based on the center of mass, stability requirement, location of all the legs, etc. This example illustrates the significance of creating topology chain when dealing with human commands.

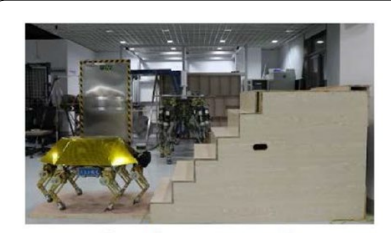

On the ground

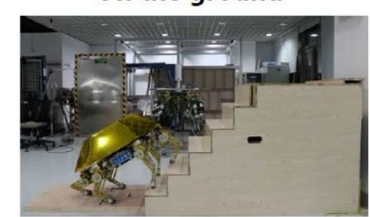

Step 2

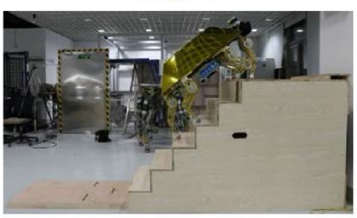

Step 6

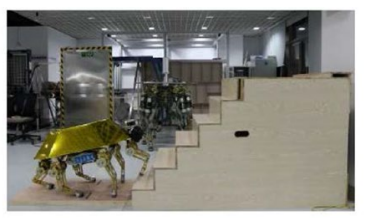

Step 1

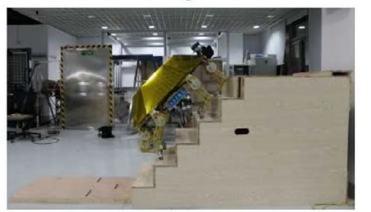

Step 5

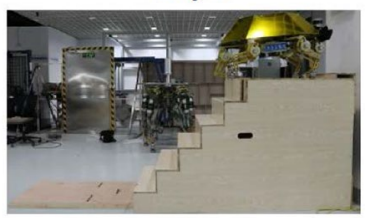

On the top
Figure 7 Climbing stairs conducted by the hexapod robot (Additional file 1) 


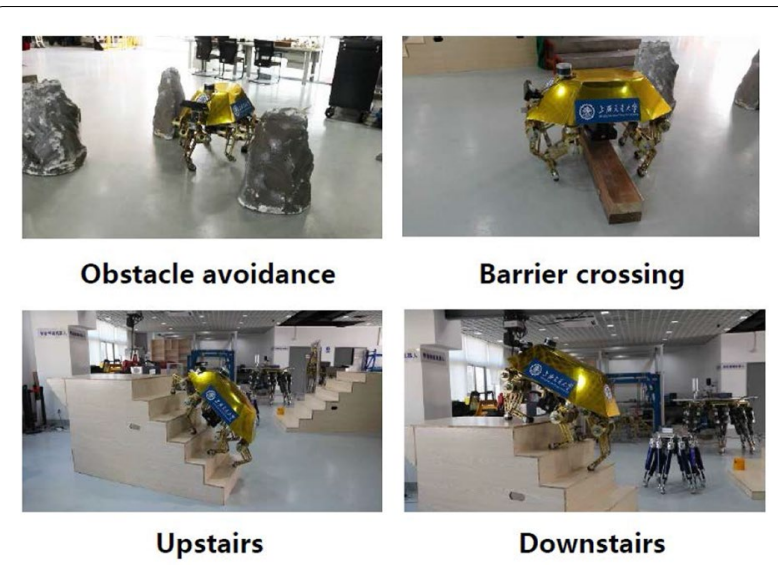

Figure 8 Screen shots of a sequence of tasks conducted by hexapod robot with a single take (Additional file 2)

\subsection{Conducting a Sequence of Tasks}

Often, the legged robot needs to accomplish a sequence of tasks given by human. In this experiment, the robot utilizes autonomous control to conducted four different tasks: obstacle avoidance, barrier crossing, climbing upstairs and downstairs in succession. In order to finish this sequence of tasks, the robot utilized the perception information acquired from camera, gyroscopes, accelerometers and force sensor which are mounted on itself. Figure 8 presents the screenshots from a singletake video. Such experiment exhibits the efficiency of autonomous control based on the framework.

\subsection{Other Complicated Tasks}

Besides the tasks mentioned above, the hexapod robots developed in our research group possess the potential capabilities to conduct other tasks. Mounted with various hands (manipulators) on the body, the hexapod robots are capable of finishing complicated tasks, including plugging the pipe, climbing steps with heavy burden, opening a door, screwing the valve, grasping the pipe and cutting the pipe, etc., as shown in Figure 9. These results demonstrate the ability to conduct various complicated tasks in unstructured environment.

\section{Conclusions}

It is still far from sufficient to establish the intelligent control system of legged robots. Therefore, continued research is needed to improve of the design and the control of legged robots. The followings are main points in this paper.

(1) The distinctive type synthesis models of legged robots mechanical design are reviewed. Based on nine

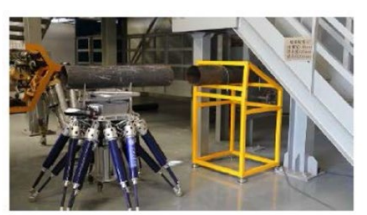

Plugging the pipe

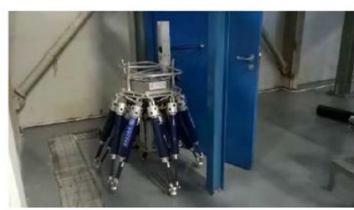

Opening the door

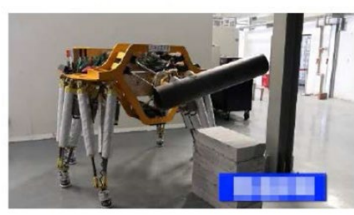

Grasping the pipe

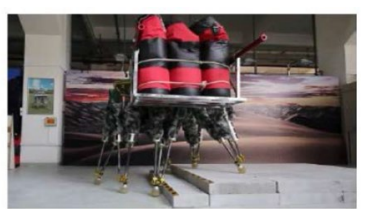

Climbing steps with heavy burden

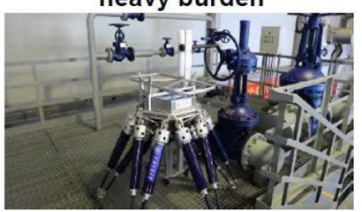

Screwing the valve

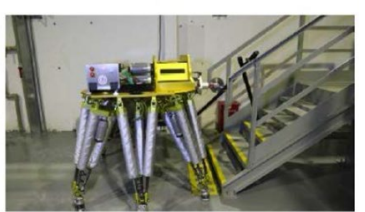

Cutting the pipe
Figure 9 Other complicated tasks accomplished by hexapod robot in unstructured environments (Additional files 3, 4, 5, 6, 7 and 8)

types of $\mathrm{G}_{F}$ sets, various hexapod robots are designed and manufactured.

(2) The control framework of legged robots is discussed, including three layers, namely data driven layer, robot behavior layer and execution layer. Each layer contains several components and are discussed in detail. To fully understand the association of the data driven layer and robot behavior layer is a key role in achieving intelligent control of the robots.

(3) Experiments on hexapod robots are implemented to execute different tasks. These results indicate the capabilities of the robots developed in our research group.

The investigation on the basic issues of type synthesis and control design for legged robots would promote the community to focus on the challenging issues and accelerate the advancement of the theoretical and practical aspects of legged robots.

\section{Additional files}

Additional file 1. Climbing stairs.

Additional file 2. Hexapod robot with a single take.

Additional file 3. Climbing steps with heavy burden.

Additional file 4. Opening the door.

Additional file 5. Plugging the pipe.

Additional file 6. Grasping the pipe.

Additional file 7. Cutting the pipe.

Additional file 8. Screwing the valve. 


\section{Authors' contributions}

LF was in charge of the whole experiment and data analysis; LF and FG wrote the manuscript. Both authors read and approved the final manuscript.

\section{Authors' information}

Ling Fang, born in 1987, is currently a post-doctoral fellow at State Key Laboratory of Mechanical System and Vibration, Shanghai Jiao Tong University, Shanghai 200240, China. Feng Gao, born in 1956, is currently a distinguished chair professor at State Key Laboratory of Mechanical System and Vibration, Shanghai Jiao Tong University, China.

\section{Competing interests}

The authors declare no competing financial interests.

\section{Funding}

Supported by National Natural Science Foundation of China (Grant No. U1613208).

\section{Publisher's Note}

Springer Nature remains neutral with regard to jurisdictional claims in published maps and institutional affiliations.

Received: 26 February 2018 Accepted: 8 June 2018

Published online: 10 July 2018

\section{References}

[1] B Siciliano, O Khatib. Springer handbook of robotics. Springer Handbook of Robotics, 2008, 56(8): 987-1008.

[2] M Raibert, K Blankespoor, G Nelson, et al. BigDog, the Rough-Terrain Quadruped Robot. IFAC Proceedings Volumes, 2008, 41(2): 10822-5.

[3] S Kuindersma, R Deits, M Fallon, et al. Optimization-based locomotion planning, estimation, and control design for the atlas humanoid robot. Autonomous Robots, 2015, 40(3): 429-455.

[4] R Bloss. Robot walks on all four legs and carries a heavy load. Industrial Robot, 2012, 39(5): 524-525.

[5] A S Boxerbaum, J Oro, G Peterson, et al. The latest generation Whegs ${ }^{\mathrm{TM}}$ robot features a passive-compliant body joint. Proceedings of the IEEE/RSJ International Conference on Intelligent Robots and Systems, F, Nice, France, 2008: 1636-1641.

[6] T B Smith, J Barreiro, D Chavez, et al. ATHLETE's feet: Multi-resolution planning for a hexapod robot. 2008, NASA. https://ti.arc.nasa.gov/m/profile/ de2smith/publications/ICAPS08-SPA-Athlete.pdf

[7] PYang, F Gao. Mechanism topology design for novel parallel-parallel hexapod robot. Proceedings of the Ukacc International Conference on Control, F. Loughborough, UK, 2014: 737-742.

[8] M Alamir, N Marchand. Numerical stabilisation of non-linear systems: Exact theory and approximate numerical implementation. European Journal of Control, 1999, 5(1): 87-97.

[9] I R Manchester, U Mettin, F lida, et al. Stable dynamic walking over uneven terrain. International Journal of Robotics Research, 2011, 30(3): 265-279.

[10] H Dai, R Tedrake. L2-gain optimization for robust bipedal walking on unknown terrain. IEEE International Conference on Robotics and Automation IEEE, Karisruhe, 2013: 3116-3123.

[11] J M Bourgeot, N Cislo, B Espiau. Path-planning and tracking in a 3D complex environment for an anthropomorphic biped robot. IEEE/RSJ International Conference on Intelligent Robots and Systems, Switzerland, 2002, 3: 2509-2514

[12] K K Hauser, T Bretl, J C Latombe, et al. Motion planning for legged robots on varied terrain. International Journal of Robotics Research, 2007, 27(11): 1325-1349.

[13] E Yoshida, C Esteves, I Belousov, et al. Planning 3-D collision-free dynamic robotic motion through iterative reshaping. IEEE Transactions on Robotics, 2008, 24(5): 1186-1198.

[14] E Luther. International federation of robotics. Acu Publishing, 2012.
[15] R J Full, D E Koditschek. Templates and anchors: neuromechanical hypotheses of legged locomotion on land. Journal of Experimental Biology, 1999, 202(Pt 23): 3325.

[16] M H Raibert, E R Tello. Legged robots that balance. MIT Press, 1986.

[17] X Meng, F Gao, Q J Ge. Number synthesis of parallel robotic mechanisms. Mechanics Based Design of Structures \& Machines, 2014, 42(2): 211-228.

[18] X Meng, F Gao, SWu, et al. Type synthesis of parallel robotic mechanisms: Framework and brief review. Mechanism \& Machine Theory, 2014, 78(4): 177-186.

[19] T S Mruthyunjaya. A computerized methodology for structural synthesis of kinematic chains: Part 2 - Application to several fully or partially known cases. Mechanism \& Machine Theory, 1984, 19(6): 497-505.

[20] D Gan, Q Liao, S D Jian, et al. Design and kinematics analysis of a new 3CCC parallel mechanism. Robotica, 2010, 28(7): 1065-1072.

[21] J M Herv. Lie group of rigid body displacements, a fundamental tool for mechanism design. Mechanism \& Machine Theory, 1999, 34(5): 719-730.

[22] Z M Bi, SY T Lang, D Zhang, et al. Integrated design toolbox for tripodbased parallel kinematic machines. Journal of Mechanical Design, 2007, 129(8): 799-807.

[23] X Kong, C M M Gosselin. Type synthesis of 3DOF spherical parallel manipulators based on screw theory. Management International Review 2004, 126(1): 7-26.

[24] F Gao, W Li, X Zhao, et al. New kinematic structures for 2-, 3-, 4-, and 5-DOF parallel manipulator designs. Mechanism \& Machine Theory, 2002, 37(11): 1395-1411.

[25] Z Huang, Qinchuan L. Type synthesis principle of minor-mobility parallel manipulators. SCIENTIA SINICA Technologica, 2002, 45(3): 241-248.

[26] Z Huang, Q C Li. Type synthesis of symmetrical lowermobility parallel mechanisms using the constraint-synthesis method. International Journal of Robotics Research, 2003, 22(1): 59-82.

[27] G Gogu. Structural synthesis of fully-isotropic translational parallel robots via theory of linear transformations. European Journal of Mechanics - $A$ / Solids, 2004, 23(6): 1021-1039.

[28] G Gogu. Mobility and spatiality of parallel robots revisited via theory of linear transformations. European Journal of Mechanics / A Solids, 2005, 24(4): 690-711.

[29] Q Jin, T L Yang. Theory for topology synthesis of parallel manipulators and its application to three-dimension-translation parallel manipulators. Journal of Mechanical Design, 2004, 126(4): 625-639.

[30] H Shen, TYang, L Z Ma. Synthesis and structure analysis of kinematic structures of 6-dof parallel robotic mechanisms. Mechanism \& Machine Theory, 2005, 40(10): 1164-1180.

[31] J Meng, G Liu, Z Li. A geometric theory for analysis and synthesis of Sub-6 DoF parallel manipulators. IEEE Transactions on Robotics, 2007, 23(4): 625-649.

[32] F Gao, J Yang, Q J Ge. Type synthesis of parallel mechanisms having the second class $G(F)$ sets and two dimensional rotations. Proceedings of the Traffic and Transportation Studies, F, Boston, USA. 2011: 623-632.

[33] F Gao, Y Zhang, W Li. Type synthesis of 3-DOF reducible translational mechanisms. Robotica, 2005, 23(3): 239-245.

[34] J Yang, F Gao, Q J Ge, et al. Type synthesis of parallel mechanisms having the first class gf sets and one-dimensional rotation. Robotica, 2011, 29(6): 895-902.

[35] J K Davidson, K H Hunt, G R Pennock. Robots and screw theory: Applications of kinematics and statics to robotics. Oxford University Press, 2004.

[36] J S Dai. An historical review of the theoretical development of rigid body displacements from Rodrigues parameters to the finite twist. Mechanism \& Machine Theory, 2006, 41(1): 41-52.

[37] J M Herve, F Sparacino. Structural synthesis of 'parallel' robots generating spatial translation. Proceedings of the International Conference on Advanced Robotics, Boston, USA, 1991: 808-813.

[38] T L Yang, A X Liu, H P Shen, et al. On the correctness and strictness of the position and orientation characteristic equation for topological structure design of robot mechanisms. Journal of Mechanisms \& Robotics, 2013, 5(2): 021009.

[39] C Shen, L Hang, TYang. Position and orientation characteristics of robot mechanisms based on geometric algebra. Mechanism \& Machine Theory, 2017, 108: 231-243

[40] F Gao, J L Yang, J Q Ge. Type synthesis of parallel robots using GF set thoery. Science Press, 2011. (in Chinese) 
[41] R Orfali, H Dan. Client/server programming with Java and CORBA. J. Wiley \& Sons, 1998.

[42] N J Nilsson. Principle of artificial intelligence. Intelligent Systems IEEE, 1982, 29(2): 2-4.

[43] A Staranowicz, G L Mariottini. A survey and comparison of commercial and open-source robotic simulator software. Proceedings of the International Conference on Pervasive Technologies Related To Assistive Environments, F, Heraklion, Crete, Greece, 2011: 56.

[44] G N Saridis. Intelligent robotic control. Automatic Control IEEE Transactions on, 1983, 28(5): 547-557.

[45] R Lumia, J Fiala, A Wavering. The NASREM robot control system standard. Robotics \& Computer Integrated Manufacturing, 1989, 6(4): 303-308.

[46] P U Lima, G N Saridis. Design of intelligent control systems based on hierarchical stochastic automata. WORLD SCIENTIFIC, 1996.

[47] R J Firby. Adaptive execution in complex dynamic worlds. Yale University, 1989.

[48] R A Brooks. A robust layered control system for a mobile robot. IEEE Journal on Robotics \& Automation, 1986, 2(1): 14-23.

[49] E Gat. Integrating planning and reacting in a heterogeneous asynchronous architecture for controlling real-world mobile robots. Proceedings of the Tenth National Conference on Artificial Intelligence, F, San Jose, California 1992: 809-815

[50] N Muscettola, P P Nayak, B Pell, et al. Remote agent: to boldly go where no Al system has gone before. Artificial Intelligence, 1998, 103(1-2): 5-47.

[51] E Gat, R P Bonnasso, R Murphy, et al. On three-layer architectures. Artificial Intelligence \& Mobile Robots, 1998: 195-210.

[52] C Breazeal, B Scassellati. Infant-like social interactions between a robot and a human caretaker. Submission, 2008, 8(1): 49-74.

[53] H Ishiguro, T Kanda, K Kimoto, et al. A robot architecture based on situated modules. Proceedings of the IEEE/RSJ International Conference on Intelligent Robots and Systems, 1999 IROS, F, Kyongju, Korea, 1999: 1617-1624.

[54] T Kanda, T Hirano, D Eaton, et al. Person identification and interaction of social robots by using wireless tags. Proceedings of the leee/rsj International Conference on Intelligent Robots and Systems, F, Las Vegas, USA, 2003: 1657-1664.

[55] CM Bishop. Pattern recognition and machine learning (information science and statistics). Springer-Verlag New York, Inc., 2006.

[56] K Kosuge, Y Hirata. Human-robot interaction. IEEE Robotics \&amp amp amp Automation Magazine, 2009, 7(6): 85-89.

[57] R R Murphy, T Nomura, A Billard, et al. Human-robot interaction. Robotics \&Automation Magazine IEEE, 2005, 17(2): 85-89.
[58] Y Lecun, Y Bengio, G Hinton. Deep learning. Nature, 2015, 521 (7553): 436-444.

[59] M J B Krieger, J B Billeter, L Keller. Ant-like task allocation and recruitment in cooperative robots. Nature, 2000, 406(6799): 992-995.

[60] A Shademan, R S Decker, J D Opfermann, et al. Supervised autonomous robotic soft tissue surgery. Science Translational Medicine, 2016, 8(337): 337 ra64.

[61] H E B Russell, L K Harbott, I Nisky, et al. Motor learning affects car-to-driver handover in automated vehicles. Science Robotics, 2016, https://doi. org/10.1126/scirobotics.aah5682.

[62] Y Moon, NY Ko, K Lee, et al. Real-time EtherCAT master implementation on Xenomai for a robot system. International Journal of Fuzzy Logic \& Intelligent Systems, 2009, 9(3): 244-248.

[63] A Koubaa. Robot operating system (ROS). Studies in Computational Intelligence, 2016, 1(6): 342-348.

[64] E Krotkov, D Hackett, L Jackel, et al. The DARPA robotics challenge finals: Results and perspectives. Journal of Field Robotics, 2018: 34.

[65] A Norton, W Ober, L Baraniecki, et al. Analysis of human-robot interaction at the DARPA Robotics Challenge Finals. The International Journal of Robotics Research, 2017, 36(5-7): 483-513.

[66] A Settimi, C Pavan, V Varricchio, et al. A modular approach for remote operation of humanoid robots in search and rescue scenarios. Proceedings of the International Workshop on Modelling and Simulation for Autonomous Systems, F, London, UK, 2014: 192-205.

[67] T Zhao, E Li, H Bian, et al. Type synthesis and analysis of rotational parallel mechanisms with a virtual continuous axis. Mechanism \& Machine Theory, 2017, 109: 139-154

[68] M Frindt, M Krefft, J Hesselbach. Structure and type synthesis of parallel manipulators. Springer Berlin Heidelberg, 2010.

[69] X Meng, F Gao. Basic problems and criteria for synthesis of robotics. International Journal of Mechanisms \& Robotic Systems, 2013, 1(1): 35-48.

[70] F Gao, X Liu, W A Gruver. Performance evaluation of two-degree-offreedom planar parallel robots. Mechanism \& Machine Theory, 1998, 33(6): 661-668.

[71] X Meng, F Gao, J Yang. The GF sets: A new kind of performance criterion of mechanisms. Proceedings of the ASME 2012 International Design Engineering Technical Conferences and Computers and Information in Engineering Conference, F, Buffalo, USA, 2012: 559-564.

[72] J X Chen. The evolution of computing: AlphaGo. Computing in Science \& Engineering, 2016, 18(4): 4-7.

\section{Submit your manuscript to a SpringerOpen ${ }^{\circ}$ journal and benefit from:}

- Convenient online submission

- Rigorous peer review

- Open access: articles freely available online

- High visibility within the field

- Retaining the copyright to your article

Submit your next manuscript at $\boldsymbol{\nabla}$ springeropen.com 